\title{
APRENDIZAJE AUTORREGULADO Y METAS QUE PERSIGUEN LOS ALUMNOS EN LA CLASE DE EDUCACIÓN FÍSICA
}

\author{
Miguel A. Barrera M. *
}

\section{RESUMEN}

Este trabajo pretende profundizar en una temática que día a día ha ido cobrando interés en el ámbito educativo, situando su contenido, motivación y autorregulación, en una de las materias del currículo escolar más desprovista de investigación y reflexión teórica respecto a cómo aprenden los estudiantes; nos referimos a la actividad física realizada al interior de la escuela (educación física). El aprendizaje autorregulado ocurre cuando el estudiante activa y mantiene conductas sistemáticamente orientadas hacia la consecución de objetivos; en este sentido, la adquisición de conocimientos, incluidos los conocimientos prácticos como lo son aquellos referidos al ejercicio físico y al deporte, implica actividades dirigidas por metas, que los estudiantes son capaces de poner en marcha, mantener y modificar.

Palabras clave: Motivación, autorregulación, actividad física.

\section{SELF-REGULATED LEARNING AND GOALS PURSUED BY STUDENTS IN THE PHYSICAL EDUCATION CLASS}

\begin{abstract}
The aim of this article is to explore an issue that has been growing interest within the scope of education day after day, placing its content, motivation and self-regulation, into one of the areas in the school curriculum lacking research and theoretical reflection on how students learn; the author refers to the physical activity carried out within the school (Physical Education). Self-regulated learning takes place when students actively and systematically maintain behaviours aimed towards the achievement of objectives. Likewise, the acquisition of knowledge, including practical skills, such as those relating to physical exercise and sport, involves goal-directed activities, which students are able to implement, maintain and modify.
\end{abstract}

Key Words: Motivation, self-regulation, physical activity.

* Doctor en Psicología educativa, Magíster en Educación Física. Universidad Católica Silva Henríquez. mbarrera@ucsh.cl 
Aprendizaje autorregulado y metas que persiguen los alumnos en la clase de educación física

\section{Introducción}

El aprendizaje autorregulado ocurre cuando el estudiante activa y mantiene conductas sistemáticamente orientadas hacia la consecución de objetivos; en este sentido, la adquisición de conocimientos, incluidos los conocimientos prácticos como lo son aquellos referidos al ejercicio físico y al deporte, implica actividades dirigidas por metas, que los estudiantes son capaces de poner en marcha, mantener y modificar. Ahora bien, al referirnos a logros y metas, entonces debemos decir que el concepto de motivación, en la actualidad, debe considerar la coordinación del sujeto para activar y dirigir sus conductas hacia metas. Tanto es así que Covington (2000) concluye que la calidad del aprendizaje como la voluntad de continuar aprendiendo depende estrechamente de una interacción entre las clases de metas sociales y académicas que los alumnos traen a la clase, las propiedades motivadoras de estas metas y las estructuras que prevalecen en las salas de clase, aspecto este último que, dada la actual situación de la educación física en gran parte de los colegios de nuestro país, resulta un factor muy relevante a tener en cuenta.

El presente trabajo, ha sido desarrollado a partir de los cimientos teóricos presentados en la Tesis doctoral de quien expone las siguientes líneas (Barrera, 2009). En estas nos enfocaremos en aquellas metas que se buscan en el contexto escolar, metas cuya finalidad es el aprendizaje de conductas que faciliten su participación en las diferentes actividades escolares; en particular, profundizaremos en aquellas que están presentes cuando los estudiantes se enfrentan a la actividad física deportiva.

$\mathrm{Al}$ respecto, muchos autores consideran que los posibles efectos positivos de las metas en la conducta no son automáticos, sino que dependen de sus propiedades: especificidad, proximidad, nivel de dificultad o grado de participación del sujeto en su formulación. Las metas especificas favorecen el aprendizaje y activan autoevaluaciones positivas con mayor probabilidad que las generales; también promueven la autoeficacia, porque los avances en la tarea son fáciles 
de conseguir. Las próximas dan lugar a una mayor motivación que las lejanas: es más fácil obtener progresos hacia ellas, y la percepción de estos avances favorece la autoeficacia (Eccles \& Wigfield, 2002). En cuanto a la dificultad de la meta, ésta condiciona el esfuerzo que el sujeto está dispuesto a invertir para alcanzar el objetivo. Sin embargo, cuando las metas son excesivamente elevadas, como lo sería en el caso de la educación física encestar I0 de I0, muchas de las actuaciones resultarán decepcionantes para el sujeto: tener fracasos reiterados a pesar de los esfuerzos debilita la autoeficacia, reduciendo la motivación.

Sabemos que, los estudiantes cuando se ven enfrentados a sus actividades, lo hacen en función de variadas preocupaciones que requieren ser asumidas y resueltas. Por tanto elucidar aquellas que se dan en los contextos escolares, es un aspecto que abordamos en las próximas líneas.

Metas que persiguen los alumnos cuando se involucran en tareas de aprendizaje

Es necesario saber que cuando los estudiantes realizan sus actividades escolares, dirigen sus esfuerzos, de manera consciente y en algunas ocasiones sin plena conciencia, al cumplimiento de variados objetivos, finalidades o metas, las cuales pasamos a exponer de manera sintetizada siguiendo a Alonso Tapia (2005b), quien a su vez las expone esquematizadamente, producto de sus propios estudios y de una serie de otros trabajos realizados recientemente:

1. Búsqueda de autonomía y control personal. La importancia de esta meta que parece responder a una necesidad básica ha sido puesta de manifiesto por DeCharms (1976) y Deci \& Ryan (I985). Esta meta se tiene presente, de modo general, cuando un estudiante decide involucrarse en una actividad por su propio interés sin que haya sido obligado por otros. Este hecho hace que resulte interesante analizar la implicancia que tiene esta meta en la práctica de ejercicio físico, ya que es ampliamente aceptado 
Aprendizaje autorregulado y metas que persiguen los alumnos en la clase de educación física

por una gran mayoría de personas que la realización de este tipo de actividad es por sí mismo motivante, que gusta y atrae a las personas. Sin embargo, sucede que cuando se ejecuta en el marco de la escuela, estamos hablando de la educación física, esta actividad ya no resulta tan interesante y atractiva para un número importante de estudiantes.

Ahora bien, este hecho se vuelve un problema mayor cuando aquellas personas que están en su entorno y que son las encargadas de su formación piensan que nada se puede hacer para ayudarles (profesores, padres). La respuesta es posible encontrarla si logramos conocer algunos factores que influyen en que se acepte realizar una actividad de Motu propio y propuesta por otros. Tres son los factores que deben tenerse en cuenta:

a. Debe percibirse que las actividades que se les proponen ofrecen la posibilidad de desarrollo personal en línea con un aumento de su propia autonomía.

b. Deben percibir que las actividades propuestas les permitan experimentar que son competentes, que sí pueden realizar las tareas asignadas.

c. Estar bien dispuesto para realizar una tarea, exige que esta se relacione con lo que a los alumnos les interesa y con lo que ellos valoran.

Por su parte, cuando una actividad es interesante para un estudiante y ve con nitidez que los ejercicios y tareas motrices que se le proponen se relacionan con aquello que a él o ella le interesa, seguramente lo que se le propone tendrá efectos positivos sobre su motivación. Esto se traduce en que la realizará con mayor frecuencia, agrado, concentración e intensidad, y le va a costar menos hacerla. Esto significa que, si queremos que nuestros estudiantes trabajen no sintiéndose obligados, será útil que los profesores(as) de educación física les mostremos la relación que las actividades motrices a realizar tienen con aquello que les interesa. Así también, resulta conveniente que investiguemos si, con lo que nosotros hacemos, les mostramos modelos en los que pueden ver que trabajar y esforzarse 
por aprender la variedad de contenidos que propone la educación física es satisfactorio y vale la pena hacerlo.

2. Búsqueda de aceptación incondicional. La importancia de esta meta, que también parece responder a una necesidad básica, ha sido puesta de manifiesto por Wentzel (1999), autora que se ha referido a ella al hablar de la importancia del "caring" -cuidado y apoyo prestado de forma afectuosa-. Ahora bien, un hecho que es habitual observar en las personas, en particular en aquellos en edad escolar, es la necesidad de tener la atención y el afecto de los demás. En varios de sus trabajos, Alonso Tapia encontró que uno de los factores que más motivan a los alumnos de diferentes niveles educativos a esforzarse por aprender es la disponibilidad que tienen los profesores para ayudarles, ya sea dentro de la clase como fuera de ella. Es bueno saber que cuando un alumno se siente aceptado pese a sus limitaciones, mayor es la motivación por aprender y también mayor es su rendimiento.

Ahora bien, aunque sentirse aceptado por parte de los adultos ayuda a la motivación de acuerdo con los datos de la investigación que ya hemos comentado anteriormente, su aporte no es demasiado alto. Este hecho sugiere que sentirse acogido es una condición necesaria pero no suficiente para que se dé la motivación por aprender. Una de las razones de este hecho es que la aceptación incondicional puede tener consecuencias positivas o negativas dependiendo de qué cosas sean las que valoran las personas que les aceptan y que, por tanto, son importantes para ellos. Si los profesores y padres valoran, por ejemplo, el hecho de obtener buenas notas, los escolares se esforzarán por la nota más que por aprender, a fin de no perder su apoyo y aceptación. Por tanto, es necesario que los profesores(as) de educación física conozcan sobre qué otros elementos actuar para ayudar a que nuestros escolares acrecienten una motivación positiva hacia el aprendizaje de conductas proclives hacia la práctica de ejercicio físico y los deportes.

3. Deseo de aprender y experimentar competencia. La importancia de esta meta ha sido puesta de manifiesto en numerosas ocasiones, pero 
consideramos básico el trabajo de Dweck \& Elliot (1983). Se puede decir que la meta que mueve a un alumno a aprender $y$ sentirse con mayor competencia, se observa cuando durante la realización de una tarea lo que parece mantener su interés y esfuerzo es la actividad misma. Sentir que se progresa en la realización de una tarea motriz, comprender lo que dice un texto o las explicaciones que da el profesor, conlleva sentimientos de placer. Sin embargo, y dado que este deseo se activa y se mantiene fundamentalmente cuando las tareas se plantean como un desafío no competitivo centrado en el aprendizaje y cuando el estudiante recibe retroalimentación, lo que puede hacerse es plantearles tareas de dificultad intermedia (ni muy fácil, ni muy difícil) y, además, que ellas sean presentadas como un problema que encierra un desafío.

4. Deseo de aprender lo que es relevante y útil. Que los estudiantes no buscan aprender cualquier cosa sino aquello que perciben como relevante y útil ha sido puesto de manifiesto por Alonso Tapia (2005a). Es común observarlos evitar el trabajo escolar sin tener en cuenta los efectos negativos que dicha actitud conlleva. Esta situación se torna problemática cuando los profesores se sienten imposibilitados de no hacer algo para modificar esta situación, dada la indiferencia absoluta que muestran estos estudiantes por las consecuencias que su actuar provoca. Si lo que se enseña se hace a partir de problemas de la vida cotidiana, poniendo de manifiesto la utilidad que tiene aprender a resolver ese tipo de problemas, la motivación por esforzarse a aprender probablemente sea mayor, especialmente si el estudiante ha interiorizado el valor que tiene la capacidad que se quiere desarrollar. Pero dado que a pesar de lo que se haga siempre existen aquellos que no tienen el mismo interés por aprender, la pregunta que emerge es qué es lo que se puede hacer para que los estudiantes perciban que a través del conocimiento de la utilidad de la tarea puedan moverse a aprender. Lo que se puede hacer es lo siguiente:

a. Enfrentarlos a problemas que les hagan sentir la necesidad de adquirir una determinada destreza o un tipo de conocimiento. 
b. Nuestro actuar debe reflejar que valoramos las competencias expresadas por nuestros estudiantes.

c. Escuchar y observar lo que ellos dicen para conectar la tarea que se les propone con lo que valoran sus amigos y/o grupo de iguales.

En consecuencia, y de manera especial, será preciso que los profesores(as) de educación física presten colaboración a los estudiantes a ver que la práctica del deporte y la realización de los variados ejercicios que se pretende que aprendan son útiles para su vida diaria, entre ellos para mantenerse sanos y saludables para enfrentarse a las variadas exigencias físicas que se solicitan. Además, y tan importante como lo anterior, es que exige coherencia entre lo que decimos respecto al valor de la práctica regular de ejercicio físico y lo que hacemos. Es decir, con nuestro ejemplo ofrezcamos modelos donde puedan aprender a valorar las distintas competencias que el programa solicita que deben adquirir.

5. Deseo de ser útily poder ayudar a los demás. La importancia de esta meta ha sido puesta de manifiesto por Wentzel (1993, 1994) y Alonso Tapia (2005a). Un hecho que se ha constatado es que saber que lo que se aprende puede servir para ayudar a los demás, aumenta el interés y el esfuerzo por aprender. Sin embargo, no todos piensan de igual manera, en particular en realidades como la chilena donde el individualismo y el valerse por sí mismo son alimentados desde diversas esferas de la realidad nacional. Siguiendo la línea expuesta en las metas anteriores, cabe preguntarse qué es lo que se puede hacer para que los estudiantes valoren el significado de ser útil y poder ayudar a los demás como una forma de motivarse por aprender. Uno de los factores que más influye en la conducta altruista es la de ser capaz de ponerse en el lugar de otro, compartiendo sentimientos y emociones. Un segundo aspecto que ve facilitada la consecución de metas altruistas en los estudiantes es cuando se refuerza en éstos los comportamientos prosociales que manifiestan. Por último, un factor que contribuye 
Aprendizaje autorregulado y metas que persiguen los alumnos en la clase de educación física - Miguel A. Barrera

a que los estudiantes valoren el poder ayudar y también esforzarse por aprender, es mostrándoles a personas que trabajan por otras de manera desinteresada, asimismo, presentando personas que se preparan para ser útiles a otros siendo felices con lo que hacen. Obviamente, si los profesores de educación física no somos un modelo en este sentido, difícilmente podemos contribuir a que valoren el hecho de esforzarse por aprender para poder ayudar. Y no lo somos, por ejemplo, si nuestros alumnos no ven que disfrutemos cuando realizamos nuestras clases o cuando se nos invita a participar de algún evento deportivo damos excusas para no participar.

6. Deseo de obtener una calificación aceptable. Distintos estudios han puesto de manifiesto la importancia de conseguir una calificación no necesariamente superior a la de los demás pero sí aceptable (Alonso Tapia y Ruiz, 2008; Grant \& Dweck, 2003). Es un hecho de la realidad que desde varios frentes (los propios estudiantes, los padres, los profesores), las calificaciones son altamente valoradas como un medio para sobresalir en la vida. Sin embargo, esta misma realidad nos muestra que existiendo un gran número de ellos que funcionan sólo por aprobar, no todos se motivan de igual manera cuando se trata de aprendizaje por comprensión e integración de ideas. La nota resulta un elemento motivante sólo cuando lo que se tiene que aprender es una tarea de baja exigencia, memorística o de aplicación mecánica de algunos procedimientos, y además cuando la tarea a realizar le provoca rechazo, es decir, cuando el alumno no tendría ningún interés en realizarlas de no ser por el incentivo que supone la calificación.

De acuerdo con lo expuesto, no parece que insistir en la evaluación y las calificaciones para estimular el interés y el esfuerzo de los alumnos por aprender sea apropiado pues, si bien puede que se preocupen más por cumplir con lo solicitado en clases, su modo de estudiar no es adecuado para conseguir un aprendizaje de calidad. Más bien habría que analizar qué factores hacen que la evaluación y las calificaciones tengan los efectos negativos descritos para actuar sobre ellos. Lo único que parece estimularles es la 
obligación de tener que aprobar. Este hecho plantea el problema de cómo utilizar la evaluación y las calificaciones justamente para motivarles a esforzarse por aprender comprendiendo y asimilando realmente lo que estudian, algo que las evaluaciones actuales justamente parecen impedir. La clave de la solución parece estar en la naturaleza misma de las actividades de evaluación. Diversos autores han comprobado que existe una discrepancia entre los tipos de aprendizaje que se desea que los estudiantes consigan y los que se evalúan habitualmente, algo que se observa de manera frecuente en las clases de educación física. En consecuencia, si se modifica la forma de evaluar en la clase de modo que incluya tareas que no puedan superarse con un aprendizaje repetitivo y mecánico, como parecen ser la mayoría de las tareas que se llevan a cabo en esta clase, tal vez sea posible aprovechar la motivación por la calificación para estimular el interés y el esfuerzo por desarrollar otras capacidades superiores.

7. Deseo de obtener recompensas externas a la tarea. Es muy común ver, en gran parte del estudiantado, que estos vuelcan sus esfuerzos en el trabajo escolar, pensando en las recompensas externas que recibirán por él, no viendo en el trabajo la posibilidad de aprender ni desarrollar sus capacidades. Esta idea se ve afianzada en algunos casos, como lo es cuando el interés inicial del alumno por realizar la tarea es muy bajo, aspecto que se ve favorecido cuando se recibe un beneficio externo; también se da en tareas que solicitan constante repetición, esta repetición hace que la tarea resulte fácil, lo que provoca satisfacción. Por último, opera el refuerzo externo cuando se requiere cierto nivel de destreza en un componente de la tarea para poder disfrutar de lo que puede proporcionar la realización de la misma. Sin embargo, motivar al alumno desde fuera de la tarea ofreciéndole recompensas, puede que produzca aumento de su esfuerzo pero no mejoría en la calidad de su trabajo, dado que la atención se vuelca sobre la recompensa y no sobre los procedimientos y estrategias que ha de poner en práctica (Deci, Koestner \& Ryan, I999).

El hecho de ofrecer recompensas en una clase o en la casa por la realización de una tarea hace que los estudiantes inicialmente 
Aprendizaje autorregulado y metas que persiguen los alumnos en la clase de educación física - Miguel A. Barrera

interesados en la misma no vuelvan a realizarla espontáneamente, excepto si se le vuelven a ofrecer, y especialmente si son tangibles. La razón parece ser que las recompensas cambian el significado que la realización de la actividad tiene para los alumnos. Estos aprenden que si ya hay una razón para la realización de la tarea -la recompensa-, no merece la pena hacerla cuando nadie ofrece una recompensa a cambio. Este sistema que se utiliza con bastante frecuencia en clase de educación física y que se observa cuando se les ofrece a los alumnos dejarlos jugar al fútbol al término de la clase si realizan las tareas motrices que el profesor o la profesora han preparado, hace que los alumnos terminen por aprender que no merece la pena hacer las cosas por sí mismas y que lo prudente es esperar que el profesor(a) les ofrezca en cada clase algo a cambio.

8. Deseo de éxito y reconocimiento público. La importancia de esta meta fue puesta de manifiesto hace tiempo por Atkinson (I964, 1974) y, desde una perspectiva ligeramente distinta, por Dweck \& Elliot (I983). Los hechos demuestran que los seres humanos necesitamos sentirnos reconocidos y valorados en lo que hacemos, tanto de modo interno como por los demás. Este deseo se manifiesta en los estudiantes en la tendencia a compararse y a presumir de los propios éxitos. De manera general estos alumnos necesitan demostrar que tienen habilidad, cuestión que hace que se la pasen pendientes de los resultados de lo que hacen. Dado que el deseo de ser valorado positivamente hace que los alumnos centren su atención en los resultados y no en los aprendizajes, con la consiguiente pérdida de interés por aprender, resulta clave hacer que centren su atención en el proceso de aprender y en las competencias que van adquiriendo por sobre la valoración que hará el resto de los resultados que obtienen.

En cuanto a las implicaciones que para la educación física y el deporte tiene esta meta, pensamos que una correcta comprensión e interpretación de la misma por parte del profesorado de educación física, permitirá que en la planificación y puesta en escena de las actividades a desarrollar en clases, consideren no solo actividades competitivas sino que cada vez se abran mayores 
espacios para la realización de actividades cooperativas que minimicen la tendencia natural a compararse entre los alumnos por sus habilidades y capacidades físicas; todo ello, como se ha dicho, contribuye a una pérdida de la valoración positiva de sí mismo. Si esto se consigue, serán más conscientes de cómo se hacen las distintas tareas y de cómo se resuelven los problemas, conciencia que aumentará su experiencia de competencia y, en consecuencia, su autoestima.

9. Deseo de no dejarse desbordar por la presión de la tarea. La importancia de esta meta fue puesta de manifiesto hace tiempo por Mandler \& Sarason (1952) mientras estaban trabajando para tratar de explicar la ansiedad ante la ejecución. Es posible que muchos de nosotros hayamos experimentado que al vernos enfrentados a una tarea, la presión que ejercen la complejidad de ella, la presencia de otras personas y el tiempo que se nos otorga para resolverla, actúen en sentido opuesto a lo que se supone que debiera ocurrir, esto es, en vez de sentirnos presionado, y queriendo abandonar, sacamos fuerza de flaqueza y al final rendimos bien. Es claro que tal comportamiento tiene que ver con la seguridad y confianza que sentimos en nosotros mismos, sin embargo, también es posible que se deba a la necesidad de evitar que la presión de la tarea nos desborde para así sentirnos dueños de manejar la situación. Lo expuesto puede que sea una realidad en algunos estudiantes, pero la inmensa mayoría observará la presión que sienten ejercida, como un problema que haría disminuir las expectativas de éxito. Dada la evidencia, lo que se pretende es aumentar la resistencia de los estudiantes para que no se sientan agobiados cuando se enfrentan a tareas con características como las arriba señaladas. Lo que procede es enseñarles formas de pensar y actuar que junto con aumentar su resistencia a la presión, reduzcan al mínimo su miedo a no ejecutar correctamente un procedimiento motriz.

10. Deseo de no dejarse presionar por el profesor. La importancia de esta meta ha sido puesta de manifiesto por Alonso Tapia (2005b). $\mathrm{Al}$ respecto, la dinámica que se da en las aulas entre estudiantes y profesor, mediada por los entornos de aprendizaje, es un lugar propicio para que se den situaciones en que los estudiantes se 
Aprendizaje autorregulado y metas que persiguen los alumnos en la clase de educación física

sientan injustamente tratados con la consiguiente pérdida de interés por aprender. En situaciones como ésta se da que el estudiante, en vez de someterse, aumenta su esfuerzo tratando de vencer la actitud del profesor que él nota como desmotivante. Es un hecho que promover en los alumnos la defensa de sus derechos ante situaciones que ellos perciben como injustas, trae aparejado un aumento de su interés y esfuerzo por aprender; también conduce a un enfrentamiento entre profesor-alumno, que para efectos de fortalecer las ganas por aprender pareciera algo inútil. Más aún si lo que el alumno observa como un trato injusto por parte del profesor, se debe más que nada, y como lo ha comunicado Alonso Tapia en varios de sus trabajos, al planteamiento del profesor(a) que les hace pensar en vez de reproducir mecánicamente lo que ha visto en clases. Aspecto este último que, si bien no es nada frecuente en clase de educación física, es una cuestión necesaria de abordar, pues va en directa relación con los aprendizajes que el programa de estudios de la educación física de nuestro país, propicia ser alcanzados por los estudiantes.

Que los estudiantes persigan esta meta, como ocurre con mucha frecuencia en las clases de educación física dado su carácter eminentemente procedimental, hacen que los estudiantes que ven la clase de educación física solo como un espacio para la recreación, entren en disputa con su profesor(a) cuando se les solicita tareas como las ejemplificadas anteriormente. Esto significa que los profesores(as) de educación física debemos observar lo que hacemos y eventualmente modificar nuestras conductas si así fuese necesario.

Se puede mejorar la situación, sin deteriorar el necesario desarrollo de la capacidad del alumno por defender sus derechos, si los profesores(as) hacemos lo siguiente:

a. si explicamos a los estudiantes nuestro formas de actuar y evaluar a la vez que les enseñamos a resolver las diferentes tareas que se dan en clases de educación física utilizando estrategias adecuadas. 
b. Si les estimulamos a mostrar sus desacuerdos de modo adecuado, reconociendo que podemos equivocarnos y que su punto de vista puede ayudarnos a mejorar nuestra enseñanza.

c. Si les escuchamos en caso de discrepancia y somos capaces de modificar nuestro punto de vista si es errado.

d. Si creemos que nuestro punto de vista es correcto y por tanto no es necesario modificarlo, entonces debemos justificar nuestro proceder dando las razones respectivas.

11. Deseo de evitar el fracaso y la valoración negativa. La importancia de esta meta fue puesta de manifiesto hace tiempo por Atkinson (I964, 1974), desde una perspectiva ligeramente distinta, por Dweck \& Elliot (1983), y por la reciente revisión de Elliot (2005). Los estudiantes que persiguen esta meta lo hacen pensando en resguardar su autoestima y su valía personal. Para tal efecto se busca evitar las consecuencias negativas que acompañan al fracaso escolar. A modo de ejemplo, es posible observar que los estudiantes no preguntan en clases por el miedo que tienen de quedar expuestos al ridículo. También es posible observar que en situaciones en que deben elegir desarrollar una tarea, eligen la más fácil pues así no quedan desprotegidos ante el fracaso. Si estos perciben que no van a poder realizar bien una tarea, tienden a evitar el esfuerzo para poner a resguardo la autoestima. El problema es que si actuamos como está ejemplificado arriba, disminuye la posibilidad de adquirir las competencias que aumentarán su autoestima. El hecho de querer evitar la valoración negativa que sigue al fracaso tiene como fundamento la preocupación por la valía personal. El efecto de la preocupación por la propia valía es negativo por varias razones. Por un lado, si tras esforzarse en una tarea los alumnos fracasan, no se puede atribuir el fracaso a la falta de esfuerzo. Solo cabe concluir que se es incompetente, lo cual resulta humillante y es motivo de rechazo.

Dado que, en la clase de educación física los estudiantes que buscan evitar el fracaso y que a su vez se les valore negativamente han ido en aumento de manera significativa, asunto que se explica básicamente por dos razones, una de ellas dice relación con el 
Aprendizaje autorregulado y metas que persiguen los alumnos en la clase de educación física - Miguel A. Barrera

explosivo aumento de los niveles de sobrepeso y obesidad que ve afectada su participación en la realización de las diferentes tareas motrices; la otra, se relaciona con la persistente actitud de una parte importante del profesorado de educación física por ejecutar en sus clases actividades centradas en el rendimiento y la competición deportiva por sobre el dominio de otras capacidades. Esta situación es posible corregirla ayudándoles a no pensar que su valía es objeto de evaluación. Asunto este que no es fácil, ya que el sistema escolar obliga a ser evaluado en variados momentos y situaciones. Lo que se puede hacer es, en la medida de lo posible, que aprendan a considerar los errores no como un indicador de falta de capacidad, sino como algo normal de lo que también se puede aprender. Por último, ayudándoles a mantener expectativas de éxito realistas, asunto este último que es posible lograr dividiendo los ejercicios en pasos que permitan controlar la dificultad que estos tienen.

En cuanto a las metas que persiguen los estudiantes cuando realizan sus tareas de aprendizaje, sostenemos a modo de hipótesis, que las actividades que realizan los profesores de Educación física en sus clases tienen altos componentes que promueven la búsqueda de rendimiento más que la de dominio, aspecto que afecta la motivación de un porcentaje importante de ellos por adherir a la práctica regular de actividad física; cuestión ésta que ha sido evidenciada desde diversos trabajos de investigación, uno de los cuales nos señala que tanto los estudiantes primarios como secundarios muestran patrones de motivación y aprendizaje más positivos cuando sus entornos escolares bacen bincapié en el dominio, entendimiento y perfeccionamiento de las babilidades y del conocimiento (Meece, Anderman, Anderman, 2006). Similar situación nos plantea Linnenbrink (2005), quien nos señala al respecto que los resultados sugieren que es necesario poner énfasis en el aprendizaje y en la comprensión del medio ambiente del aula. Como las metas personales de acercamiento-ejecución son inadaptativas, es importante que el medio ambiente del aula, se centre en el dominio y que la competición se base en grupos que compitan en lugar de individuos que compitan. Una posible forma de hacerlo sería a través de la creación de un 
aula basada en los principios de las metas de dominio, donde los profesores utilizan variadas y significativas tareas, y proporcionan oportunidades para que los estudiantes tomen decisiones y orienten su propio aprendizaje, reconociendo a la vez que los estudiantes pueden aprender y mejorar.

Por su parte Covington (2000), refiriéndose al tema de la competición a nivel escolar nos indica que cuando las metas son competitivas, se tiende a continuar esforzándose mientras se tiene éxito, pero nadie quiere seguir si el resultado es la vergüenza y las recriminaciones a uno mismo. Así también, la introducción de incentivos competitivos, de cualquier clase o en cualquier cantidad, no provoca un aumento, sino una disminución, del logro escolar, al menos en las tareas complejas y significativas.

Al profundizar en lo que los teóricos de metas de logro han ido produciendo, podemos manifestar que éstos se centran en las razones o intenciones que tienen los estudiantes para interesarse, elegir, y persistir en diferentes actividades de aprendizaje. El trabajo realizado por estos, ha desembocado en que se han puesto a la vista del lector especializado variados conceptos, por también variados autores, como lo expone Meece et al. (2006). Conceptos duales tales como aprendizaje - rendimiento, implicancia en la tarea - implicancia en el yo, enfocado en el dominio - enfocado en la capacidad, enfocado en la tarea - enfocado en la capacidad. Si bien ha sido larga la discusión por determinar el destino de estos términos, la mayoría de los investigadores, varios de ellos ya citados en este texto, han concluido que estos pares de metas tienen varios elementos en común lo que permitiría que ellos fuesen tratados como elaboraciones teóricas conceptualmente similares, dando lugar a lo que se ha denominado como "orientaciones de meta".

En las próximas líneas, y habiendo optado por utilizar los conceptos dominio y rendimiento, precisamos el significado de cada uno de ellos. Respecto a la orientación de meta de dominio ésta se define en términos de una atención centrada en desarrollar las propias capacidades, dominar una nueva babilidad, intentar lograr alguna tarea desafiante, y tratar de 
Aprendizaje autorregulado y metas que persiguen los alumnos en la clase de educación física - Miguel A. Barrera

entender los materiales de aprendizaje (Meece et al., 2006. Op. cit.). Por su parte, una orientación de meta de rendimiento representa una atención centrada en demostrar una alta capacidad en relación a los demás, en esforzarse por ser mejor que los otros, y en utilizar los niveles sociales de comparación para emitir juicios respecto a la capacidad y el rendimiento (ídem). Ahora bien, los teóricos de metas, en general están de acuerdo en que las metas de dominio son más productivas que las metas de rendimiento. A pesar de que estas últimas, en algunas situaciones, carecen de efecto negativo, como por ejemplo lo sería en cursos altamente competitivos en la universidad, donde los alumnos son clasificados en las típicas curvas de rendimiento. Dado esto se recomienda utilizarlas como un complemento a las metas de acercamiento al dominio (Brophy, 2005), cuestión esta que es refutada por Covington (2000), quien manifiesta que la competición bajo cualquier condición es negativa.

Sin embargo, desde hace tiempo se han venido articulando nuevos enfoques para entender conductas que se suscitan en el aula y que no logran ser explicitadas con claridad por las orientaciones señaladas recientemente; nos referimos a la orientación a la evitación, cuyo foco está en relación con el deseo de evitar el fracaso y la valoración negativa que se puede obtener de los demás (Elliot \& Covington, 200I; Midgley, Kaplan \& Middleton, 200I; Eccles \& Wigfield, 2002). Aspectos estos últimos que se han profundizado, en cuanto a su esclarecimiento y significado, por los trabajos de Alonso Tapia (2005b, c; 2008), el cual nos manifiesta que también esto depende del grado en que las tareas y la actividad escolar en su conjunto se perciben como inútiles y, muy especialmente, por el grado en que los estudiantes carecen de las estrategias y aptitudes adecuadas para enfrentarse, por un lado, a la presión generada por el contexto real de las clases definido por el orden en el que han de trabajar, y por otro, a los límites de tiempo dentro de los que deben informar de su aprendizaje como al desaliento producido por la disposición de ánimo de los profesores(as).

En cuanto a las dos primeras orientaciones de metas expuestas en párrafos anteriores, en el último tiempo los investigadores 
han manifestado no sólo la necesidad de hacer una distinción entre ellas, sino que además someterlas a una permanente revisión (Harackiewics, Barron, Pintrich, Elliot \& Thrash, 2002). De las primeras, de rendimiento, se han clasificado o distinguido entre metas de aproximación al rendimiento y metas de evitación del rendimiento. En cuanto a las segundas opera similar distinción, es decir, metas de aproximación al dominio y metas de evitación al dominio; ambas se persiguen con la finalidad de mantener cierto equilibrio en la estimación de su persona.

Evitación y acercamiento son los polos de un mismo continuum que como nos dicen Elliot \& Covington (200I), son fundamentales a la hora de intentar explicar e interpretar la conducta de las personas ante determinados eventos, como sería el caso de estudiantes que no desean ser vistos como incompetentes ante sus compañeros dada la dificultad que tienen para la realización de los ejercicios que se disponen en clase de Educación física.

Reiteramos que son muchas las referencias de la literatura especializada en que se indica que los estudiantes demuestran patrones de logro más positivos cuando centran su atención en metas de dominio. Por su parte las metas de rendimiento están asociadas a estrategias de aprendizaje superficial. Sin embargo, estos patrones no se mantienen en todos los estudios, y los investigadores sugieren marcar la diferencia entre las formas de acercamiento y evitación de las metas de rendimiento (Harackiewicz et al., 2002). Aspecto que aborda Stevenson (2006), en una investigación cuyo propósito fue examinar la viabilidad del modelo sociocognitivo estudiado con el fin de determinar si puede ayudar a los investigadores a comprender mejor las motivaciones y los patrones de actividad física informados por los individuos. El estudio pretendió distinguir la relación de varios constructos, entre ellos las metas dentro de la participación en actividades físicas de alta y moderada intensidad. Para tal efecto se propuso revisar el modelo incorporando un marco de meta de logro de $2 \times 2$ y colocando la competencia como un antecedente para la adopción de una meta. Los resultados avalaron la hipótesis de 
Aprendizaje autorregulado y metas que persiguen los alumnos en la clase de educación física - Miguel A. Barrera

que las metas de acercamiento al dominio entregan una mediación parcial para la influencia positiva de la competencia en la actividad física intensa, y una mediación parcial para la influencia positiva de la competencia y de la teoría incremental (la inteligencia es maleable) en la actividad física moderada. En cuanto a las metas de evitación de rendimiento, estas entregaron una supresión parcial para la influencia positiva de la competencia percibida; y mediaron la influencia negativa de la teoría de la identidad (la inteligencia está fija). Se destaca que aquellos individuos que adoptaron metas de evitación de rendimiento fueron los mismos que mantuvieron creencias de entidad, ya que estas personas también poseen una baja autonomía relativa.

A pesar de lo expuesto, los estudios actuales destacan la necesidad de reconocer que los estudiantes pueden adoptar múltiples metas de forma simultánea (Kaplan, Middleton, Urdan \& Midgley, 2002; Brophy, 2005), las que relativamente se adaptan, en menor o mayor grado, al aprendizaje; esto quiere decir que éstas no se dan en forma aislada, sino que están en permanente relación y que cambian con el tiempo (Boekaerts, 1999; Boekaerts \& Corno, 2005; Alonso Tapia, 2005b). Sin embargo, a pesar de la ingente investigación en el tema, aún no está claro cuál combinación de metas se adapta mejor a cada grupo de estudiantes, tareas de logro y contextos de aprendizaje (Midgley et al., 200I).

Al dar una mirada retrospectiva respecto a los planteamientos teóricos acerca de las metas que tienen presentes los sujetos cuando eligen una actividad, permanecen en ella o dan muestras de que su conducta al implicarse en ellas toma ciertos rumbos y determinadas intensidades, en un comienzo se pensaba que el comportamiento en ambientes como el académico estaba influido por el deseo al éxito y a su vez miedo al fracaso, esto se daba principalmente por las consecuencias tanto positivas como negativas que podía tener para la autoimagen (Alonso Tapia, 2005a).

Si bien la mayoría de los trabajos apoya con evidencias las metas descritas arriba, y que estas evidencias favorecen en forma abrumadora la hipótesis de la teoría de metas, la cual indica que las diferentes 
razones para lograr aproximación y evitación, influyen en la calidad del rendimiento esforzándose a través de mecanismos autorreguladores (Covington, 2000); existen algunos investigadores que piensan que existen otras metas que permiten explicar de mejor manera los procesos motivacionales que se dan en la sala de clases. Entre ellos tenemos las que nos exponen Covington (2000) y Eccles y Wigfield (2002), quienes revisando varios trabajos relativos al tema, verifican que éstos ponen de manifiesto la existencia de otras metas que para los estudiantes serían muy importantes y que además estarían influyendo de diversas maneras en el trabajo escolar; estas serían las metas sociales. Una de ellas, la meta por ser respetado y aceptado por los demás -asunto que es promovido por el estilo de vida imperante en nuestro país- nos parece ser una interesante veta de estudio y que eventualmente podría estar dando cuenta de una posible respuesta a la problemática presentada en este trabajo; nos referimos a aquella relacionada con las dificultades que se tendrían, en el caso escolar chileno, para el aprendizaje de conductas proclives a una vida física activa y saludable.

Al concluir, debemos manifestar que la incipiente, pero en aumento, profesionalización de la práctica de la actividad físico-deportiva en nuestro país, ha convertido en objeto de interés creciente el desarrollo de estrategias motivacionales que faciliten la intervención; tanto si se trata de aumentar el interés por adherir a los diferentes programas deportivos, como por mejorar la motivación por el aprendizaje de destrezas y/o habilidades deportivas.

Cuando nos referirnos al interés por la práctica educativo-física en los colegios, lo hacemos pensando en que la participación en actividades físico-deportivas al interior del sistema educativo formal, es vista crecientemente como una necesidad que debe ser satisfecha dado el carácter de la educación física como parte integrante del currículo oficial en el sistema escolar chileno; así como también, pensando en que lo que debe aprenderse en esta clase es un contenido educativo que se encuentra claramente definido en los programas de estudio respectivo (Mineduc, Programas de estudios de educación física, 200I). 
Aprendizaje autorregulado y metas que persiguen los alumnos en la clase de educación física

\section{Bibliografía}

Alonso Tapia, J. (2005a). Motivaciones, Expectativas y Valores relacionados con el Aprendizaje. Artículo publicado por el Ministerio de educación, cultura y deporte dentro del libro: Premios Nacionales a la Investigación Educativa, Madrid.

Alonso Tapia, J. (2005b). Motivar en la escuela, motivar en la familia. Claves para el aprendizaje. Madrid: Morata.

Alonso Tapia, J. (2005c). Motivación para el aprendizaje: la perspectiva de los alumnos. Publicado en: Ministerio de Educación y Ciencia. La orientación escolar en centros educativos (pp. 209-242). Madrid: MEC.

Alonso Tapia, J. \& Ruiz, M. A. (2008). Motivation, self-regulation and academic achievement: A study on their relationships and educational implications coming from the structural and predictive validity of the MEVA questionnaire. Artículo enviado para su publicación.

Atkinson, J. W. (1964). An introduction to motivation. Princeton, NJ: Van Nostrand.

Atkinson, J. W. (1974). The mainspring of achievement oriented activity. En J.W. ATKINSON (Ed.), Motivation and achievement (pp. I3-4I). Nueva York: Wiley.

Barrera, M. (2009). Educación para la salud: valoración de un programa de intervención para el desarrollo de la motivación hacia la práctica de ejercicio físico. Tesis doctoral no publicada, Facultad de Psicología, Universidad Autónoma de Madrid.

Boekarts, M. (1999). Self-regulated learning: where we are today. International Journal of Educational Research, 3I, 445-457

Boekaerts, M. \& Corno, L. (2005). Self-Regulation in the Classroom: A Perspective on Assessment and Intervention. Applied Psychology: an International Review, 54 (2), 199-231

Brophy, J. (2005). Goal Theorists should move on from performance goals. Educational Psychologist, 40(3), 167-176

Covington, M.V. (2000). Goal theory, Motivation, and School achievement: An integrative Review. Annual Review of Psychology, 5I, I7I-200.

Decharms, R. (1976). Enhancing motivation: Change in the classroom. Nueva York: Irvington. Citado por: J. Alonso Tapia (2005), Motivar en la escuela, Motivar en la familia. Madrid, Morata.

Deci, E. L. \& Ryan, R. M. (1985). Intrinsic motivation and self-determination in 
human behavior. Plenium Press, Nueva York. Citado por J. Alonso Tapia (2005), Motivar en la escuela, motivar en la familia. Madrid, Morata.

Deci, E. L.; Koestner, R \& Ryan, R. M. (I999). A meta-analytic review of experiments examining the effects of extrinsic rewards on intrínsic motivation. Psychological Bulletin, I25, 6, 627-688.

Dweck, C. \& Elliot, E. S. (1983). Achievement motivation. En P. H. Mussen (Editor general) y E. M. Hetherington (ed. del vol.), Handbook of child psychology. Vol. IV: Social and personality development (pp. 643-69I). Nueva York, Wiley.

Eccles, J.S. \& Wigfield, A. (2002). "Motivational Beliefs, Values and Goals". Annual Review of Psychology, 53, I09-I32.

Elliot, A. J. (2005). A conceptual history of achievement goal construct. En A.J. Elliot y C. Dweck (Eds.), Handbook of competence and motivation (pp. 52-72). Nueva York: Guilford.

Elliot, A. J. \& Covington, M. V. (200I). Aproach and Avoidance motivation. Educational Psychology Review, I3 (2), 73-92.

Grant, H. \& Dweck, C. (2003). Clarifying achievement goals and their impact. Lournal of Personality and Social Psychology, 85 (3), 54I-553.

Harackiewics, J.M.; Barron, K.E.; Pintrich, P.R.; Elliot, A.J. \& Thrash, T.M. (2002). Revision of Achievement Goal Theory: Necessary and Illuminating. Journal of Educational Psychology, 94(3), 638-645.

Kaplan, A.; Middleton, M.; Urdan, T. \& Midgley, C. (2002). Achievement goals and goal structures. In C. Midgley (Ed.), Goals, goal structures, and patterns of adaptative learning (pp. 2I-53). Mahwah, NJ: Erlbaum

Linnenbrink, E. A. (2005). The Dilemma of Performance-Approach Goals: The Use of Multiple Goal Contexts to Promote Students' Motivation and Learning. Journal of Educational Psychology, 97, (2), 197-213

Mandler, G. \& Sarason, S.B. (I952). A study of anxiety and learning. Journal of Abnormal and Social Psychology, 47, I66I73.

Meece, J.; Anderman, E. \& Anderman, L. (2006). Classroom goal structure, student motivation, and academic achievement. Annual Review of Psychology, 57, 487-503.

Midgley, C.; Kaplan, A. \& Middleton, M. (200I). Performance-Approach Goals: Good For What, For Whom, Under What Circumstances, and At What Cost? Journal of Educational Psychology, 93 (I), 77-86.

Stevenson, S. (2006). Understanding strenuous and moderate intensity exercise participation and motivation: a social-cognitive perspective. A Thesis in 
Aprendizaje autorregulado y metas que persiguen los alumnos en la clase de educación física

- Miguel A. Barrera

sport Psychology. Submitted to the graduate of texas tech University in partial.

Wentzel, K. R. (1993). Motivation and achievement in early adolescence: The role of multiple classroom goals. Journal of Early Adolescence, 23, 4-20.

Wentzel, K. R. (1994). Relations of Social Goal Pursuit to Social Acceptance, Classroom Behavior, and Perceived Social Support. Journal of Educational Psychology, 86, 173-I82.

Wentzel, K. R. (1999). Social Influences in school adjustment: Commentary. Educational Psychology, 34,I, 59-69. Citado por J. Alonso Tapia (2005), Motivar en la escuela, motivar en la familia, Morata, Madrid. 